\title{
Black holes in bi-scalar extensions of Horndeski theories
}

\author{
Christos Charmousis, ${ }^{a, b}$ Theodoros Kolyvaris, ${ }^{c}$ Eleftherios Papantonopoulos ${ }^{d}$ \\ and Minas Tsoukalas ${ }^{e}$ \\ ${ }^{a}$ Laboratoire de Physique Théorique (LPT), Université Paris-Sud, CNRS UMR 8627, \\ F-91405 Orsay, France \\ ${ }^{b}$ Laboratoire de Mathématiques et Physique Théorique (LMPT), CNRS UMR 6083, \\ Université Francois Rabelais, \\ Tours, France \\ ${ }^{c}$ Instituto de Física, Pontificia Universidad Católica de Valparaíso, \\ Casilla 4950, Valparaiso, Chile. \\ ${ }^{d}$ Department of Physics, National Technical University of Athens, \\ Zografou Campus GR 157 73, Athens, Greece. \\ ${ }^{e}$ Centro de Estudios Científicos, \\ Casilla 1469, Valdivia, Chile \\ E-mail: christos.charmousis@th.u-psud.fr, theodoros.kolyvaris@ucv.cl, \\ lpapa@central.ntua.gr, minasts@cecs.cl
}

ABSTRACT: We study certain bi-scalar-tensor theories emanating from conformal symmetry requirements of Horndeski's four-dimensional action. The former scalar is a Galileon with shift symmetry whereas the latter scalar is adjusted to have a higher order conformal coupling. Employing techniques from local Weyl geometry certain Galileon higher order terms are thus constructed to be conformally invariant. The combined shift and partial conformal symmetry of the action, allow us to construct exact black hole solutions. The black holes initially found are of planar horizon geometry embedded in anti de Sitter space and can accommodate electric charge. The conformally coupled scalar comes with an additional independent charge and it is well-defined on the horizon whereas additional regularity of the Galileon field is achieved allowing for time dependence. Guided by our results in adS space-time we then consider a higher order version of the BBMB action and construct asymptotically flat, regular, hairy black holes. The addition of the Galileon field is seen to cure the BBMB scalar horizon singularity while allowing for the presence of primary scalar hair seen as an independent integration constant along-side the mass of the black hole.

KEYwords: Classical Theories of Gravity, Black Holes

ARXIV EPRINT: 1404.1024 


\section{Contents}

1 Introduction 1

2 Setup 4

3 Planar black holes with a cosmological constant and a Maxwell field $\quad 7$

3.1 Planar stealth solution $\quad 8$

$\begin{array}{lll}3.2 & \text { Electric solution with a flat horizon } & 9\end{array}$

4 Introducing a conformally coupled scalar $\phi \quad 10$

$5 \quad$ Introducing linear dependence and regularity $\quad 11$

$\begin{array}{lll}5.1 & \text { Regular planar black hole } & 11\end{array}$

$\begin{array}{ll}\text { 5.2 Constructing an asymptotically flat hairy black hole } & 13\end{array}$

6 Conclusions 16

$\begin{array}{ll}\text { A Variations } & 17\end{array}$

\section{Introduction}

There has been a renewed interest in modified gravity theories due to cosmological observations pointing towards an accelerating Universe [1,2]. Gravity theories resulting from the Horndeski Lagrangian [3] belong to a general class of scalar-tensor theories which have been under intense investigation recently. Apart from their mathematical generality, there are two special reasons why Hornedski theories are attractive. First of all they are consistent and technically manageable. This stems from the fact that they give second-order field equations. Secondly, a subset of these scalar-tensor theories of modified gravity share a classical Galilean symmetry around flat space-time (Galileon theories) or around curved space-time (Generalized Galileon theories) [4-6].

In four dimensions, the most general Galileon theory with second order field equations was given a long time ago by Horndeski [3], and can be written in the simpler form presented in [7],

$$
\begin{aligned}
S_{\text {Horndeski }}[\chi, g]= & \int d^{4} x \sqrt{-g}\left[K(\chi, X)-G_{3}(\chi, X) \mathcal{E}_{1}\right. \\
& \left.+G_{4}(\chi, X) R+G_{4, X} \mathcal{E}_{2}+G_{5}(\chi, X) G_{\mu \nu} \nabla^{\mu} \nabla^{\nu} \chi-\frac{G_{5, X}}{6} \mathcal{E}_{3}\right]
\end{aligned}
$$

where $X=-\frac{1}{2}(\nabla \chi)^{2}, \mathcal{E}_{n}=n ! \nabla_{\left[\mu_{1}\right.} \nabla^{\mu_{1}} \chi \cdots \nabla_{\left.\mu_{n}\right]} \nabla^{\mu_{n}} \chi$ and commas denote differentiation i.e. $G_{4, X}=\frac{\partial G_{4}}{\partial X}$. Note that the functions $G_{i}$ appearing in (1.1) are in general functions 
of the scalar field $\chi$ and its kinetic term $X$. In a nutshell Horndeski or Galileon theory is the most general scalar-tensor theory with second order field equations in four-dimensional space-time.

Few local solutions are known, due to the complexity of this higher order theory (1.1). One way of approach is via known black hole solutions of higher dimensional metric theories. It has been known since a long time, that Lovelock theory (for a review see [8-10]) yields several Gallileon terms by Kaluza-Klein compactifications [11-14]. Following this path, four dimensional analytic black hole solutions of Horndeski theories were first found by KaluzaKlein reduction of higher dimensional Lovelock theory [15]. Interestingly the higher order solutions cloak naked singularities of lower order Einstein-dilaton theories, by introducing a novel event horizon. They are however not asymptotically flat. At about the same time a nice no hair argument was introduced concerning asymptotically flat solutions for Gallileons [16]. There it was argued, under some generic hypotheses, that static, spherically symmetric black hole solutions for the gravity-galileon coupled system could not sustain primary scalar hair, for vanishing boundary condition at infinity (see also [18]). To prove this the shift-symmetry of the galileon action and the regularity of diffeomorphic invariant quantities at the horizon was used. Ways to circumvent the no hair argument have been discussed in $[17,38]$ and we will exploit these here explicitly.

One of the elegant terms appearing in the Horndeski Lagrangian is the derivative coupling of the scalar field to the Einstein tensor

$$
I=\int d^{4} x \sqrt{-g}\left[\frac{R}{16 \pi G}-\left(g^{\mu \nu}-G(\chi) G^{\mu \nu}\right) \nabla_{\mu} \chi \nabla_{\nu} \chi\right],
$$

pictured here alongside with the canonical kinetic term. The above, gives second order field equations due essentially to the divergence free property of the Einstein tensor. The Einstein scalar tensor term is one of the Fab 4 terms [19, 20], having interesting implications on the cosmological constant problem. Furthermore, on cosmological backgrounds, this term with the function $G(\chi)$ constant, leads to an accelerated expansion without the need of any scalar potential, as noted for the first time in [21]. The presence of this coupling in the Lagrangian gives second-order field equations [22] as part of a Kaluza Klein reduction of Einstein-Gauss-Bonnet theory. These features attracted much interest in inflationary cosmology [23, 24], particle production after inflation [25] and also late-time cosmology [26-28].

Local solutions for the action (1.2) and with the coupling function $G$ constant were discussed in several recent papers [29-33]. Spherically symmetric black hole solutions which are asymptotically anti-de Sitter were found. They all rely on switching off the primary hair integration constant by imposing a specific geometric Anzatz. This bifurcates the no hair argument of [16] since it allows the scalar field to be non-trivial. Unfortunately this is not enough to completely evade singular behaviour of the scalar field and solutions are generically singular for the derivative of the scalar field on the horizon. This is after all a common problem in theories with a scalar field coupled to gravity [34, 35]. To circumvent the problem of regularity of local solutions one can break shift symmetry of the scalar field by introducing a mass term for the scalar field [36, 37]. Another way to remedy this 
problem, while keeping shift-symmetry, [38], is to introduce an additional, mild, linear dependence in the time coordinate for the scalar field (see also the recent generalization of [39]). This yields an additional integration constant while the shift symmetry is essential in keeping the field equations time-independent and consistent for a static space-time Anzatz. This permits asymptotically flat (or de-Sitter) solutions and crucially gives regular scalar tensor black holes [38]. Although the role of time dependence is essential for regularity, the physical significance associated to time dependence, as a genuine scalar hair charge, is not as yet understood. An important question arises: is it possible to generate an asymptotically flat black hole with genuine primary scalar hair? This is one of the important questions we will treat in this article.

Towards this end we will consider theories of the type (1.2) where the coupling function $G(\chi)$ will be non trivial.

The strategy we will follow will be to upgrade the coupling of the scalar-tensor (1.2) interaction $G$, to full conformal invariance using results from Weyl geometry. This will effectively introduce a second scalar field and we will thus consider a bi-scalar-tensor theory. We will then look for black hole solutions in close analogy to a scalar field conformally coupled to gravity [34, 35].

Gravity theories having conformal invariance have many advantages. Conformally invariant actions seem to play an important role in early universe cosmology [40-45] and black hole physics [46]. Additionally it has been shown that the holographic renormalisation procedure in four-dimensional General Relativity (GR) can be achieved by adding a topological invariant term, which in turn gives an on shell action equal to conformal gravity [47]. On the other hand it has also been proven that starting from conformal gravity and requiring appropriate boundary conditions an equivalence with Einstein GR can be made [48]. Concerning local solutions in scalar tensor theories, adding a non-minimally coupled term of the scalar field to gravity, respecting conformal invariance, is the only coupling allowing for black hole geometries [34, 35]. However, the resulting solutions are problematic since the scalar field blows up at the event horizon. This pathology can be cured by adding a cosmological constant alongside with a self-interaction term of the scalar field [49-55]. Another question we will successfully treat in this article will be to look for regular asymptotically flat black holes of the BBMB type with primary hair.

Recently it has been proven [56], that in four dimensional scalar tensor theories, the only combination which respects conformal symmetry for a single scalar and has second order field equations, comes in the form of the the well known action [57]

$$
\int d^{4} x \sqrt{-g}\left[-\frac{1}{2} \nabla_{\mu} \phi \nabla^{\mu} \phi-\frac{1}{12} \phi^{2} R-\alpha \phi^{4}\right] .
$$

The full case for a single scalar theory in arbitrary dimension is discussed in [58]. Despite the fact that the discussion so far has been in the context of a single scalar case, we can also include higher numbers of scalars in our theory. A wide class of actions that consider a multi-scalar version of Horndeski's theory has been proposed in [59]. These theories do not possess full generality [60], but in Minkowski space-time they are the most general ones [61]. Full conformal invariance in these theories can be found by applying the method 
of Ricci gauging [62]. In this way an efficient method has been presented, where at least for the bi-scalar case we can have the most general Horndeski action (1.1) promoted to an action having a full conformal invariance that avoids Ostrogradski ghosts

$$
\begin{aligned}
S_{\text {local }}[\tilde{\phi}, \tilde{g}]= & \int d^{4} x \sqrt{-\tilde{g}}\left[K(\tilde{\phi}, \tilde{X})-G_{3}(\tilde{\phi}, \tilde{X}) \tilde{\mathcal{E}}_{1}\right. \\
& \left.+G_{4}(\tilde{\phi}, \tilde{X}) \tilde{R}+G_{4, \tilde{X}}(\tilde{\phi}, \tilde{X}) \tilde{\mathcal{E}}_{2}+G_{5}(\tilde{\phi}, \tilde{X}) \tilde{G}_{\mu \nu} \tilde{\nabla}^{\mu} \tilde{\nabla}^{\nu} \tilde{\phi}-\frac{G_{5, \tilde{X}}(\tilde{\phi}, \tilde{X})}{6} \tilde{\mathcal{E}}_{3}\right],
\end{aligned}
$$

where the composite fields $\tilde{\phi}=\phi / \pi, \tilde{g}_{\mu \nu}=\pi^{2} g_{\mu \nu}$ have the following transformation properties $g_{\mu \nu} \rightarrow \Omega(x)^{2} g_{\mu \nu}, \pi \rightarrow \pi / \Omega(x), \phi \rightarrow \phi / \Omega(x)$.

The work and our main results are organized as follows. In section 2 we will set up our bi-scalar tensor theory explaining how the scalar field coupled to the Einstein tensor can be transformed to a fully conformal invariant term with the addition of an extra scalar field $\phi$. In section 3 we will introduce a cosmological constant and an electromagnetic field and look for static black hole solutions. They will end up being of planar horizon sections and will thus necessitate a negative cosmological constant. In section 4 we will allow for the scalar field $\phi$ to be coupled conformally to gravity and find the according planar black hole. In section 5 in order to render the solutions obtained in the previous sections regular we will allow for the time dependence of the scalar field. This will lead us to eventually consider a different coupling function $G$ non conformally invariant but tailored to the lower order BBMB action [34, 35]. Then for this theory we will obtain two asymptotically flat black hole solutions of spherical symmetry where the BBMB scalar $\phi$ will be regular and have genuine primary scalar hair. Finally in section 6 are our conclusions and in the appendix we give some technical details.

\section{Setup}

Before we set up our theory we will review the black hole solutions resulting from a scalar field conformally coupled to gravity with an action given by (1.3) supplemented by an Einstein-Hilbert term with a cosmological constant.

The field equations are,

$$
\begin{aligned}
G_{\mu \nu}+\Lambda g_{\mu \nu} & =8 \pi T_{\mu \nu}, \\
\square \phi & =\frac{1}{6} R \phi+4 \alpha \phi^{3},
\end{aligned}
$$

where the energy-momentum tensor is given by

$$
T_{\mu \nu}=\partial_{\mu} \phi \partial_{\nu} \phi-\frac{1}{2} g_{\mu \nu} g^{\alpha \beta} \partial_{\alpha} \phi \partial_{\beta} \phi+\frac{1}{6}\left[g_{\mu \nu} \square-\nabla_{\mu} \nabla_{\nu}+G_{\mu \nu}\right] \phi^{2}-g_{\mu \nu} \alpha \phi^{4},
$$

and $\square \equiv g^{\mu \nu} \nabla_{\mu} \nabla_{\nu}$. Since (1.3) is invariant under conformal transformations

$$
g_{\mu \nu} \rightarrow \Omega^{2}(x) g_{\mu \nu}, \quad \phi \rightarrow \Omega^{-1}(x) \phi,
$$


the stress tensor is traceless and as a consequence, the scalar curvature is constant

$$
R=4 \Lambda \text {. }
$$

Adopting a particular spherically symmetric ansatz for the metric

$$
d s^{2}=-f(r) d t^{2}+\frac{1}{f(r)} d r^{2}+r^{2} d \Omega^{2},
$$

because of the relation (2.5) we get,

$$
f(r)=-\frac{\Lambda}{3} r^{2}+1+\frac{c_{1}}{r}+\frac{c_{2}}{r^{2}} .
$$

The $1 / r^{2}$ term appearing in (2.7) is sourced by the presence of the scalar field which plays a similar role to an EM field.

Solving the full system of equations (2.1) and (2.2) the constants $c_{1}$ and $c_{2}$ are specified and we obtain the solution

$$
d s^{2}=-\left[-\frac{\Lambda}{3} r^{2}+\left(1-\frac{G M}{r}\right)^{2}\right] d t^{2}+\left[-\frac{\Lambda}{3} r^{2}+\left(1-\frac{G M}{r}\right)^{2}\right]^{-1} d r^{2}+r^{2} d \Omega^{2}
$$

this is known as the MTZ hairy black hole [49-52]. It is important to note that for a solution to exist a consistency relation should hold connecting the constants of the theory $\alpha=-\frac{2}{9} \pi \Lambda G$. We note here that the presence of the cosmological constant in the action from which the field equations (2.1) and (2.2) result makes the scalar field regular on the horizon, hiding irregularities of the scalar field behind the horizon. The black hole, not having an independent integration constant associated to the scalar field has secondary hair.

We will follow a similar strategy to find black hole solutions in Horndeski theory (1.1) including in particular the term

$$
\sqrt{-g} G(\chi) G^{\mu \nu} \nabla_{\mu} \chi \nabla_{\nu} \chi
$$

appearing in (1.2). We will fix the coupling function $G(\chi)$ and add additional terms in order to achieve conformal invariance. We will construct our conformally invariant action in such a way that it encodes various conformal weights for one of the scalars [63], through the use of Weyl Geometry.

Let us suppose that the scalar field $\chi$ has conformal weight $w_{\chi}$ or equivalently that it transforms as

$$
\chi \rightarrow \Omega^{w_{\chi}} \chi,
$$

and the metric has the usual transformation

$$
g_{\mu \nu} \rightarrow \Omega^{2} g_{\mu \nu} .
$$

Now let us introduce a second scalar field that transforms as

$$
\phi \rightarrow \Omega^{-1} \phi .
$$


In order to construct a conformally invariant action we need to promote the metric $g_{\mu \nu}$ to $\tilde{g}_{\mu \nu}=\phi^{2} g_{\mu \nu}$ which is by construction conformally invariant and modify the covariant derivative that acts on $\chi$ as

$$
D_{\mu} \chi=\nabla_{\mu} \chi-\frac{w_{\chi}}{2} \chi \partial_{\mu} \ln \phi^{-2}
$$

Promoting the metric to the tilded one guarantees that they will be conformally invariant [56, 58] (see also [64] for an alternative way to construct conformally coupled scalar theories. This is done by introducing a four-rank tensor, that transforms covariantly under local Weyl rescallings). Furthermore the coupling function $G(\chi)$ has to take the form

$$
G(\chi)=\chi^{-2}
$$

It is easy to check that $D_{\mu} \chi \rightarrow \Omega^{w_{\chi}} D_{\mu} \chi$, which justifies the choice for the function $G(\chi)$ of $(2.14){ }^{1}$

If we now want to make a conformally invariant action using the Einstein tensor then one choice is the following

$$
\begin{aligned}
& \sqrt{-\tilde{g}} G(\chi) \tilde{G}^{\mu \nu} D_{\mu} \chi D_{\nu} \chi \\
& =\sqrt{g} \chi^{-2}\left[G^{\mu \nu} \nabla_{\mu} \chi \nabla_{\nu} \chi+2 w_{\chi} \frac{\chi}{\phi} G^{\mu \nu} \nabla_{\mu} \chi \nabla_{\nu} \phi+\left(w_{\chi} \frac{\chi}{\phi}\right)^{2} G^{\mu \nu} \nabla_{\mu} \phi \nabla_{\nu} \phi\right. \\
& +4 \phi^{-2} \nabla^{\mu} \phi \nabla^{\nu} \phi \nabla_{\mu} \chi \nabla_{\nu} \chi+6 w_{\chi} \phi^{-3} \chi \nabla^{\kappa} \phi \nabla_{\kappa} \phi \nabla^{\mu} \chi \nabla_{\mu} \phi \\
& +3\left(w_{\chi} \frac{\chi}{\phi}\right)^{2}\left(\nabla^{\mu} \phi \nabla_{\mu} \phi\right)^{2}-\phi^{-2} \nabla^{\kappa} \phi \nabla_{\kappa} \phi \nabla^{\mu} \chi \nabla_{\mu} \chi \\
& -2 \phi^{-1} \nabla^{\mu} \nabla^{\nu} \phi \nabla_{\mu} \chi \nabla_{\nu} \chi+2 \phi^{-1} \square \phi \nabla^{\mu} \chi \nabla_{\mu} \chi \\
& -4 w_{\chi} \frac{\chi}{\phi^{2}} \nabla^{\mu} \nabla^{\nu} \phi \nabla_{\mu} \chi \nabla_{\nu} \phi+w_{\chi} \frac{\chi}{\phi^{2}} \square \phi \nabla^{\mu} \chi \nabla_{\mu} \phi \\
& \left.-2 \phi^{-1}\left(w_{\chi} \frac{\chi}{\phi}\right)^{2} \nabla^{\mu} \nabla^{\nu} \phi \nabla_{\mu} \phi \nabla_{\nu} \phi+2 \phi^{-1}\left(w_{\chi} \frac{\chi}{\phi}\right)^{2} \square \phi \nabla^{\mu} \phi \nabla_{\mu} \phi\right] \text {, }
\end{aligned}
$$

which by construction is conformally invariant. The $\tilde{G}^{\mu \nu}$, refers to the Einstein tensor constructed using the rescaled metric $\tilde{g}_{\mu \nu}$ and its inverse. A choice that simplifies the above expression is $w_{\chi}=0$. Setting $\Psi=\ln (\chi)$, we have:

$$
\begin{aligned}
& \mathcal{L}=\sqrt{-\tilde{g}} G(\chi) \tilde{G}^{\mu \nu} D_{\mu} \chi D_{\nu} \chi \\
& =\sqrt{g}\left[G^{\mu \nu} \nabla_{\mu} \Psi \nabla_{\nu} \Psi+4 \phi^{-2} \nabla^{\mu} \phi \nabla^{\nu} \phi \nabla_{\mu} \Psi \nabla_{\nu} \Psi-\phi^{-2} \nabla^{\kappa} \phi \nabla_{\kappa} \phi \nabla^{\mu} \Psi \nabla_{\mu} \Psi\right. \\
& \left.\quad-2 \phi^{-1} \nabla^{\mu} \nabla^{\nu} \phi \nabla_{\mu} \Psi \nabla_{\nu} \Psi+2 \phi^{-1} \square \phi \nabla^{\mu} \Psi \nabla_{\mu} \Psi\right]
\end{aligned}
$$

and the action is shift symmetric in $\Psi$. This term which is conformally invariant will be our higher order ingredient for our bi-scalar-tensor theory.

The field equations resulting from the variation of (2.16) with respect to $g_{\mu \nu}, \Psi$ and $\phi$ are given in the following.

\footnotetext{
${ }^{1}$ See [63] for a general discussion on Weyl geometry and conformal invariance.
} 
- Field equation for the scalar field $\Psi$ :

$$
\begin{aligned}
- & 2 G^{\mu \nu} \nabla_{\mu} \nabla_{\nu} \Psi-8 \nabla_{\mu}\left(\phi^{-2} \nabla^{\mu} \phi \nabla^{\nu} \phi \nabla_{\nu} \Psi\right)+2 \nabla_{\mu}\left(\phi^{-2} \nabla^{\kappa} \phi \nabla_{\kappa} \phi \nabla^{\mu} \Psi\right) \\
& -4 \phi^{-2} \nabla^{\mu} \nabla^{\nu} \phi \nabla_{\mu} \Psi \nabla_{\nu} \phi+4 \phi^{-1} R_{\mu \nu} \nabla^{\mu} \phi \nabla^{\nu} \Psi+4 \phi^{-1} \nabla^{\mu} \nabla^{\nu} \phi \nabla_{\mu} \nabla_{\nu} \Psi \\
& +4 \phi^{-2} \square \phi \nabla^{\mu} \phi \nabla_{\mu} \Psi-4 \phi^{-1} \square \phi \square \Psi=\mathcal{E}_{\Psi} .
\end{aligned}
$$

- Field equation for the scalar field $\phi$ :

$$
\begin{aligned}
& -8 \phi^{-3} \nabla^{\mu} \phi \nabla^{\nu} \phi \nabla_{\mu} \Psi \nabla_{\nu} \Psi-8 \nabla^{\mu}\left(\phi^{-2} \nabla^{\nu} \phi \nabla_{\mu} \Psi \nabla_{\nu} \Psi\right)+2 \phi^{-3} \nabla^{\kappa} \phi \nabla_{\kappa} \phi \nabla^{\mu} \Psi \nabla_{\mu} \Psi \\
& +2 \nabla_{\kappa}\left(\phi^{-2} \nabla^{\kappa} \phi \nabla^{\mu} \Psi \nabla_{\mu} \Psi\right)+4 \phi^{-2} \nabla^{\mu} \nabla^{\nu} \phi \nabla_{\mu} \Psi \nabla_{\nu} \Psi-4 \phi^{-2} \square \phi \nabla^{\mu} \Psi \nabla_{\mu} \Psi \\
& -4 \phi^{-3} \nabla^{\mu} \phi \nabla^{\nu} \phi \nabla_{\mu} \Psi \nabla_{\nu} \Psi+4 \phi^{-3} \nabla^{\nu} \phi \nabla_{\nu} \phi \nabla^{\mu} \Psi \nabla_{\mu} \Psi-4 \phi^{-2} \nabla^{\mu} \phi \nabla^{\nu} \Psi \nabla_{\mu} \nabla_{\nu} \Psi \\
& +4 \phi^{-2} \square \Psi \nabla^{\mu} \phi \nabla_{\mu} \Psi-2 \phi^{-1}(\square \Psi)^{2}+2 \phi^{-1} \nabla_{\mu} \nabla_{\nu} \Psi \nabla^{\mu} \nabla^{\nu} \Psi+2 \phi^{-1} R^{\mu \nu} \nabla_{\mu} \Psi \nabla_{\nu} \Psi=\mathcal{E}_{\phi}
\end{aligned}
$$

- Metric field equations:

$$
\begin{aligned}
- & \frac{1}{2} g_{\mu \nu} G^{\alpha \beta} \nabla_{\alpha} \Psi \nabla_{\beta} \Psi+2 G_{(\mu}{ }^{\lambda} \nabla_{\nu)} \Psi \nabla_{\lambda} \Psi+\frac{1}{2} R \nabla_{\mu} \Psi \nabla_{\nu} \Psi-\frac{1}{2} R_{\mu \nu} \nabla^{\alpha} \Psi \nabla_{\alpha} \Psi \\
& +\frac{1}{2} g_{\mu \nu}\left((\square \Psi)^{2}-\nabla_{\alpha} \nabla_{\beta} \Psi \nabla^{\alpha} \nabla^{\beta} \Psi-R_{\alpha \beta} \nabla^{\alpha} \Psi \nabla^{\beta} \Psi\right)+\nabla_{\mu} \nabla^{\alpha} \Psi \nabla_{\nu} \nabla_{\alpha} \Psi-\square \Psi \nabla_{\mu} \nabla_{\nu} \Psi \\
& +R_{\mu \nu}{ }^{\beta} \nabla_{\alpha} \Psi \nabla_{\beta} \Psi-2 g_{\mu \nu} \phi^{-2} \nabla^{\alpha} \phi \nabla^{\beta} \phi \nabla_{\alpha} \Psi \nabla_{\beta} \Psi+8 \phi^{-2} \nabla^{\kappa} \phi \nabla_{\kappa} \Psi \nabla_{(\mu} \phi \nabla_{\nu)} \Psi \\
& +\frac{1}{2} g_{\mu \nu} \phi^{-2} \nabla^{\kappa} \phi \nabla_{\kappa} \phi \nabla^{\lambda} \Psi \nabla_{\lambda} \Psi-\phi^{-2} \nabla_{\mu} \phi \nabla_{\nu} \phi \nabla^{\kappa} \Psi \nabla_{\kappa} \Psi-\phi^{-2} \nabla^{\kappa} \phi \nabla_{\kappa} \phi \nabla_{\mu} \Psi \nabla_{\nu} \Psi \\
& +g_{\mu \nu} \phi^{-1} \nabla^{\alpha} \nabla^{\beta} \phi \nabla_{\alpha} \Psi \nabla_{\beta} \Psi-4 \phi^{-1} \nabla_{(\mu} \nabla^{\kappa} \phi \nabla_{\nu)} \Psi \nabla_{\kappa} \Psi+2 \nabla_{\kappa}\left(\phi^{-1} \nabla_{(\mu} \phi \nabla_{\nu)} \Psi \nabla^{\kappa} \Psi\right) \\
& -\nabla_{\alpha}\left(\phi^{-1} \nabla^{\alpha} \phi \nabla_{\mu} \Psi \nabla_{\nu} \Psi\right)-g_{\mu \nu} \phi^{-1} \square \phi \nabla^{\alpha} \Psi \nabla_{\alpha} \Psi+2 \phi^{-1} \nabla_{\mu} \nabla_{\nu} \phi \nabla^{\alpha} \Psi \nabla_{\alpha} \Psi \\
& +2 \phi^{-1} \square \phi \nabla_{\mu} \Psi \nabla_{\nu} \Psi-2 \nabla_{(\mu}\left(\phi^{-1} \nabla_{\nu)} \phi \nabla^{\beta} \Psi \nabla_{\beta} \Psi\right)+g_{\mu \nu} \nabla_{\alpha}\left(\phi^{-1} \nabla^{\alpha} \phi \nabla^{\beta} \Psi \nabla_{\beta} \Psi\right)=\mathcal{E}_{\mu \nu}
\end{aligned}
$$

As can be seen from the above expressions we have labeled the variation of (2.16) with respect the scalar fields $\Psi, \phi$ and the metric $g_{\mu \nu}$, as $\mathcal{E}_{\Psi}, \mathcal{E}_{\phi}$ and $\mathcal{E}_{\mu \nu}$ respectively. It can be seen that the trace of (2.19) is identically zero by virtue of the scalar field equation (2.18). Despite that these expressions are long and complicated, we will see that they can be tractable. In the following sections we will solve the equations (2.17)-(2.19) for various cases.

\section{Planar black holes with a cosmological constant and a Maxwell field}

Consider the following action

$$
S=\int d^{4} x \sqrt{-g} \frac{R-2 \Lambda}{16 \pi G_{N}}-\gamma \int d^{4} x \sqrt{-g} \frac{1}{16 \pi} F^{\mu \nu} F_{\mu \nu}+\beta \int d^{4} x \sqrt{-\tilde{g}} \tilde{G}^{\mu \nu} D_{\mu} \Psi D_{\nu} \Psi,
$$

where $\gamma$ is a dimensionless coupling constant used to switch on and off the Maxwell field, and the last term is by construction conformally invariant (section 2). The field equations for $\Psi$ and $\phi$ are respectively (2.17) and (2.18), while for the metric we have

$$
H_{\mu \nu}=\frac{1}{16 \pi G_{N}}\left(G_{\mu \nu}+\Lambda g_{\mu \nu}\right)+\gamma\left(\frac{1}{32 \pi} g_{\mu \nu} F^{\alpha \beta} F_{\alpha \beta}-\frac{1}{8 \pi} F_{\mu}{ }^{\alpha} F_{\nu \alpha}\right)+\beta \mathcal{E}_{\mu \nu}=0 .
$$


The metric ansatz we consider is the following

$$
d s^{2}=-f(r) d t^{2}+\frac{1}{f(r)} d r^{2}+r^{2} d \Omega_{\kappa}^{2},
$$

where

$$
d \Omega_{\kappa}^{2}=\frac{1}{1-\kappa x^{2}} d x^{2}+x^{2} d y^{2} .
$$

Taking the trace of (3.2) and using the field equation for $\phi$, we get $R=4 \Lambda$ from which we get,

$$
f(r)=\kappa+\frac{c_{1}}{r}+\frac{c_{2}}{r^{2}}-\frac{\Lambda}{3} r^{2} .
$$

Although conformal symmetry nicely (seems to) close in to a solution, the above is not in general a solution for the theory (3.1).

\subsection{Planar stealth solution}

With the inclusion of the cosmological constant (we set $\gamma=0$ ), we consider the metric function with a flat horizon $\kappa=0$

$$
f(r)=\frac{r^{2}}{\lambda^{2}}-\frac{m}{r},
$$

where $\Lambda=-3 / \lambda^{2}$ Then from $H_{r}{ }^{r}=0$ we get

$$
H_{r}{ }^{r}=0=>\left(\phi+r \phi^{\prime}\right)\left(3 m \phi^{\prime}+r^{2} \Lambda\left(\phi+r \phi^{\prime}\right)\right)=0 .
$$

If the first bracket is zero, then the field equations are satisfied with

$$
\begin{aligned}
\phi(r) & =\frac{c_{0}}{r}, \\
\Psi(r) & =\tilde{C}_{2}+\tilde{C}_{1} \ln \left(r^{3 / 2}+\sqrt{r^{3}-m^{2} \lambda^{2}}\right) .
\end{aligned}
$$

Notice that the scalar $\phi$ in (3.8) is regular everywhere apart from the origin and we have a scalar charge $c_{0}$. The scalar $\Psi$ is regular up to and including the horizon at $r=r_{h}$, where $f\left(r_{h}\right)=0$. Its derivative however is divergent and the scalar $\Psi$ is imaginary for $r<r_{h}$. Calculating the on shell action, particularly for the $\sqrt{-\tilde{g}} \tilde{G}^{\mu \nu} D_{\mu} \Psi D_{\nu} \Psi$ term, we can see that it is actually zero and therefore regular on shell. This irregularity of the scalar field encountered in similar solutions [29-33] can be completely remedied by including time dependence in the manner of [38] as we will see in a forthcoming section.

Now if the second bracket is zero then the field equations are satisfied with

$$
\begin{aligned}
\phi(r) & =\frac{C_{0}}{\left(-3 m-r^{3} \Lambda\right)^{1 / 3}}, \\
\Psi(r) & =C_{2}+\frac{2 C_{1}}{3 \sqrt{\Lambda}} \ln \left[2\left(r^{3 / 2} \Lambda+\sqrt{\Lambda} \sqrt{r^{3} \Lambda+3 m}\right)\right] .
\end{aligned}
$$

Here however, the solution (3.10) for the scalar field $\phi$ blows up at the horizon. If we want to have regular behaviour of the scalar field on the horizon then the scalar charge $C_{0}$ has to be zero. In this case the action (3.1) (with $\gamma=0$ ) has a local solution with one regular scalar field. Note that in this case the derivative coupling of the scalar field to the Einsten tensor is not any more conformally invariant. 


\subsection{Electric solution with a flat horizon}

We now switch back on the Maxwell field $(\gamma=1)$. The field equations for the metric are

$$
H_{\mu \nu}=\frac{1}{16 \pi G_{N}}\left(G_{\mu \nu}+\Lambda g_{\mu \nu}\right)+\frac{1}{32 \pi} g_{\mu \nu} F^{\alpha \beta} F_{\alpha \beta}-\frac{1}{8 \pi} F_{\mu}{ }^{\alpha} F_{\nu \alpha}+\beta \mathcal{E}_{\mu \nu}=0 .
$$

From the Maxwell equation we obtain

$$
\begin{aligned}
\nabla_{\mu} F^{\mu \nu} & =0, \\
A(r) & =-\frac{Q}{r} .
\end{aligned}
$$

From the trace of the field equations for $\kappa=0$ we find that the metric function is

$$
f(r)=\frac{c_{1}}{r}+\frac{c_{2}}{r^{2}}-\frac{\Lambda}{3} r^{2} .
$$

In this case the equations are satisfied only if $c_{2}=0$, or if $c_{2}=Q^{2} G_{N}$. If we choose $c_{2}=0$ then the electric charge $Q$ must also be zero, so we end up to the previous solution that we have already discussed. So $c_{2}=Q^{2} G_{N}$ is the only choice. Setting this value for $c_{2}$, from the $H_{r}^{r}=0$ equation we get

$$
H_{r}{ }^{r}=0=>\left(\phi+r \phi^{\prime}\right)\left(-3 r^{2} c_{1} \phi^{\prime}+Q^{2} G_{N}\left(\phi-3 r \phi^{\prime}\right)+r^{4} \Lambda\left(\phi+r \phi^{\prime}\right)\right)=0 .
$$

Choosing the first bracket to be zero we obtain

$$
\phi(r)=\frac{c_{0}}{r} .
$$

Substituting the value of $\phi$ back to the field equations we can solve for $\Psi^{\prime}$

$$
\Psi^{\prime}(r)=\frac{r^{3 / 2} C_{1}}{\sqrt{3 r c_{1}+4 Q^{2} G_{N}} \sqrt{-3 r c_{1}-3 Q^{2} G_{N}+r^{4} \Lambda}} .
$$

Because of the shift symmetry we only need $\Psi^{\prime}$ and $\Psi^{\prime \prime}$ since only these expressions appear in the field equations. If we calculate the value of $\sqrt{-\tilde{g}} \tilde{G}^{\mu \nu} D_{\mu} \Psi D_{\nu} \Psi$ we get once again zero as a result and therefore the fact that $\Psi^{\prime}$ is divergent on the horizon is a somewhat milder iregularity.

Now if the second bracket is zero we find that the solution for $\phi$ is

$$
\phi(r)=\frac{c_{0} r^{1 / 3}}{\left(-3 r c_{1}-3 Q^{2} G_{N}+r^{4} \Lambda\right)^{1 / 3}},
$$

and again

$$
\Psi^{\prime}(r)=\frac{r^{3 / 2} C_{1}}{\sqrt{3 r c_{1}+4 Q^{2} G_{N}} \sqrt{-3 r c_{1}-3 q^{2} G_{N}+r^{4} \Lambda}} .
$$

The introduction of a Maxwell field fixes the value of $c_{2}$ in (3.5) to be proportional to the charge. However, as it happens in the case of a constant coupling constant [29-33] the scalar fields are not regular on the horizon. It seems that another scale is needed in order to hide irregularities behind the black hole horizon. The on-shell value of $\sqrt{-\tilde{g}} \tilde{G}^{\mu \nu} D_{\mu} \Psi D_{\nu} \Psi$ is again vanishing. 


\section{Introducing a conformally coupled scalar $\phi$}

In the previous section we saw that the scalar field $\phi$ is either regular or irregular on the horizon depending on the branch we choose (3.16). Since we want to eventually close in on BBMB type solutions $[34,35]$ we will now assume that the scalar field $\phi$ has a conformal coupling to gravity. We consider therefore following action

$$
\begin{aligned}
S= & \int d^{4} x \sqrt{-g}\left[\frac{R-2 \Lambda}{16 \pi G_{N}}-\frac{1}{2} g^{\mu \nu} \nabla_{\mu} \phi \nabla_{\nu} \phi-\frac{1}{12} \phi^{2} R-\alpha \phi^{4}-\frac{1}{16 \pi} F^{\mu \nu} F_{\mu \nu}\right] \\
& +\beta \int d^{4} x \sqrt{-\tilde{g}} \tilde{G}^{\mu \nu} D_{\mu} \Psi D_{\nu} \Psi .
\end{aligned}
$$

Then the field equations for the metric become

$$
H_{\mu \nu}=\frac{1}{16 \pi G_{N}}\left(G_{\mu \nu}+\Lambda g_{\mu \nu}\right)-T_{\mu \nu}^{(\phi)}+\frac{1}{32 \pi} g_{\mu \nu} F^{\alpha \beta} F_{\alpha \beta}-\frac{1}{8 \pi} F_{\mu}{ }^{\alpha} F_{\nu \alpha}+\beta \mathcal{E}_{\mu \nu}=0,
$$

where

$$
T_{\mu \nu}^{(\phi)}=\frac{1}{2} \nabla_{\mu} \phi \nabla_{\nu} \phi-\frac{1}{4} g_{\mu \nu} \nabla^{\alpha} \phi \nabla_{\alpha} \phi+\frac{1}{12}\left[g_{\mu \nu} \square-\nabla_{\mu} \nabla_{\nu}+G_{\mu \nu}\right] \phi^{2}-\frac{1}{2} g_{\mu \nu} \alpha \phi^{4},
$$

and the field equation for the scalar field $\phi$ changes accordingly from (2.18) to

$$
\square \phi-\frac{1}{6} \phi R-4 \alpha \phi^{3}+\beta \mathcal{E}_{\phi}=0 .
$$

Setting again $\kappa=0$ we find the metric function

$$
f(r)=\frac{c_{1}}{r}+\frac{c_{2}}{r^{2}}-\frac{\Lambda}{3} r^{2}
$$

while the Maxwell field reads,

$$
A(r)=-\frac{Q}{r} .
$$

Now if we choose the scalar field to be $\phi(r)=\frac{c_{0}}{r}$, then the equation $H_{t}{ }^{t}-H_{r}{ }^{r}=0$ is automatically satisfied. We can now take the $H_{t}{ }^{r}-H_{x}^{x}=0,(x$ is a coordinate in the base manifold whose curvature $\kappa$ we have set to zero) and solve for $\Psi^{\prime}$

$$
\begin{aligned}
& H_{t}{ }^{t}-H_{x}^{x}=0=>-3 r^{2} c_{2}+3\left(Q^{2} r^{2}+2 \pi c_{0}^{2}\left(r c_{1}+2 c_{2}\right)\right) G_{N} \\
& -2 \pi \beta G_{N}\left(9 r^{2} c_{1}^{2}+4 c_{2}\left(9 c_{2}+r^{4} \Lambda\right)+6 c_{1}\left(7 r c_{2}+r^{2} \Lambda\right)\right) \Psi^{\prime 2} \\
& -4 \pi r \beta\left(3 r c_{1}+4 c_{2}\right) G_{N}\left(-3\left(r c_{1}+c_{2}\right)+r^{4} \Lambda\right) \Psi^{\prime} \Psi^{\prime \prime}=0=> \\
& \Psi^{\prime}(r)= \pm \sqrt{\frac{3 r^{2} c_{2}-\left(r^{2}\left(3 Q^{2}-2 \pi r \beta C_{1}\right)+\pi c_{0}^{2}\left(3 r c_{1}+4 c_{2}\right)\right) G_{N}}{2 \pi \beta\left(3 r c_{1}+4 c_{2}\right) G_{N}\left(-3\left(r c_{1}+c_{2}\right)+r^{4} \Lambda\right)}} .
\end{aligned}
$$

Plugging back the solution for $\Psi$ (the sign of $\Psi$ is irrelevant according to the field equations) we have the following constraint for the integration constant $c_{2}$

$$
c_{2}=\left(Q^{2}+8 \pi \alpha c_{0}^{4}\right) G_{N}
$$


This is a consistency relation between the constants and it is analogue to the consistency relations that appear in the usual conformally coupled scalar field solutions, [49-52]. Similar to what we have seen in the previous sections, the value of $\sqrt{-\tilde{g}} \tilde{G}^{\mu \nu} D_{\mu} \Psi D_{\nu} \Psi$ is vanishing. This solution is analogous to the planar black hole found in $[65,66]$. There it was found that in order to support a planar MTZ black hole [49-52], one needed to include two axionic fields. Here the same role is played by the Galileon $\Psi$ although note that the space-time metrics are quite different.

The above solution may not be the most general, since there was no systematic way to find $\phi$. We started from an obvious ansatz for $\phi$ which at the end turns out to be a correct one.

Additionally if we start from

$$
\phi(r)=\frac{c_{0} r^{1 / 3}}{\left(-3 r c_{1}-3 Q^{2} G_{N}+r^{4} \Lambda\right)^{1 / 3}}
$$

and we set $\alpha=0$ and $c_{2}=Q^{2} G_{N}$ we see that

$$
\Psi(r)= \pm \frac{r^{3 / 2 \sqrt{C 1-\frac{2 \beta c_{0}^{2}\left(3 r c_{1}+4 Q^{2} G_{N}\right)}{r^{1 / 3}\left(-3 r c_{1}-3 Q^{2} G_{N}+r^{4} \Lambda\right)^{2 / 3}}}}}{2 \beta \sqrt{3 r c_{1}+4 Q^{2} G_{N}} \sqrt{-3 r c_{1}-3 Q^{2} G_{N}+r^{4} \Lambda}}
$$

solves the equations.

Again we observe irregularity of the scalar fields on the black hole horizon, while also the value of $\tilde{G}^{\mu \nu} D_{\mu} \Psi D_{\nu} \Psi$ is once again zero.

\section{Introducing linear dependence and regularity}

In this section in order to address the irregularity of the galileon field on the horizon we will introduce a time dependent scalar field in the manner described in [38]. As before, we will first study the case of a $\Psi$-dependent derivative coupling (2.16). We will then, using the construction ideas of [38], extend the action to include a particular form of energy momentum tensor. In this way we will obtain asymptotically flat and regular solutions.

\subsection{Regular planar black hole}

Start as before with (3.1) without an electromagnetic field $(\gamma=0)$. For the metric we consider the general planar ansatz $\kappa=0$,

$$
d s^{2}=-h(r) d t^{2}+\frac{1}{f(r)} d r^{2}+r^{2} d \Omega^{2},
$$

where

$$
d \Omega^{2}=d x^{2}+x^{2} d y^{2} .
$$

Consider now that the scalar field has also a linear dependence in time, $\Psi(t, r)=q t+$ $\psi(r)$ [38, 67-69]. The field equations, due to the shift symmetry of the field $\Psi$ will still be ODE's. Furthermore, the field equation $H_{t r}=0$ is now non trivial and controls the 
flux of the scalar field $\Psi$ which is time dependent while the metric is forced to be static. Verifying this equation actually also kills the scalar field equation for the $\Psi$ field. From the latter equation, apart from the obvious solution $\Psi=$ const, we get two other possible solutions for $\phi$

$$
\phi=\frac{m}{r},
$$

as before (and regular away from $r=0$ ) and

$$
\phi=\frac{m}{(r h(r))^{1 / 3}} .
$$

We reject the latter for it will be singular whenever $h(r)=0$. We can then immediately solve for $H_{t t}=0$ to obtain,

$$
f(r)=\frac{r^{2}}{\lambda^{2}}-\frac{m}{r},
$$

and then inputting the result in the $H_{r r}$ component we obtain $h(r)=f(r)$. Finally the $H_{\theta \theta}=0$ equation gives us $\Psi(t, r)$ to be

$$
\Psi(t, r)=q t \pm \int \frac{\sqrt{q^{2}+\frac{C}{l^{2}} h(r)}}{h(r)} d r .
$$

The solution we found in section 3 is simply obtained for $q=0$. Going to EddingtonFinkelstein (EF) coordinates

$$
v=t+\int \frac{d r}{f(r)},
$$

one finds as usual a regular future chart

$$
d s^{2}=-f(r) d v^{2}+2 d v d r+r^{2} d \Omega^{2} .
$$

Applying the same transformation for $\psi$ gives

$$
\psi(v, r)=q v+\frac{C}{l^{2}} \int \frac{d r}{q \pm \sqrt{q^{2}+\frac{C}{l^{2}} h(r)}}
$$

which is regular at the future horizon for $\psi$ only for the plus branch of (5.6). The minus sign in the denominator of (5.9) is excluded, since it can cause infinities. Hence by including a time dependent scalar field $\psi$ we obtain a regular planar black hole solution. Note that the scalar $\psi$ still has a light like singularity for $v \rightarrow \infty$ but that is independent of the black hole solution and the derivative of $\psi$ appearing in the action is constant.

It is straightforward to switch on the electric charge in the action and obtain the electric version of the solution with $\psi(t, r)=q t+\psi(r)$. The metric has the previous form (3.15) with $\phi=c_{0} / r$ but the scalar field $\psi(r)$ is quite more involved. The regularity mechanism works in the same way. 


\subsection{Constructing an asymptotically flat hairy black hole}

Up to now we have obtained solutions with a negative cosmological constant and a planar horizon. Our action (3.1) was constructed so as to have conformal symmetry in the higher order term (2.16). We would obviously like to extend our results to get solutions in asymptotically flat space-time. In order to do we will use the insight gained in the previous sections and consider a slightly different action tailoring it to the construction method of [38]. Consider therefore the following action,

$$
S=S_{0}+S_{1}
$$

where

$$
S_{0}=\int d x^{4} \sqrt{-g}\left[\zeta R+\eta\left(-\frac{1}{2}(\partial \phi)^{2}-\frac{1}{12} \phi^{2} R\right)\right]
$$

and

$$
S_{1}=\int d x^{4} \sqrt{-g}\left(\beta G_{\mu \nu} \nabla^{\mu} \Psi \nabla^{\nu} \Psi-\gamma T_{\mu \nu} \nabla^{\mu} \Psi \nabla^{\nu} \Psi\right),
$$

where $T_{\mu \nu}\left(\phi, g_{\mu \nu}\right)$ is precisely the energy momentum tensor of a scalar field $\phi$ conformally coupled to gravity,

$$
T_{\mu \nu}=\frac{1}{2} \nabla_{\mu} \phi \nabla_{\nu} \phi-\frac{1}{4} g_{\mu \nu} \nabla_{\alpha} \phi \nabla^{\alpha} \phi+\frac{1}{12}\left(g_{\mu \nu} \square-\nabla_{\mu} \nabla_{\nu}+G_{\mu \nu}\right) \phi^{2} .
$$

Here, only the part of the action $S_{0}$ multiplied with $\eta$ is conformally invariant. Moreover contrary to the previous cases we do not need to set any conformal weight for $\Psi$, which means that not only $\beta$ is dimension-full, but also $\gamma$. The reason for considering such an action is the following. Consider the $\Psi$ field equation (obtained from (5.12)) which due to shift symmetry can be nicely written as a current conservation equation,

$$
\nabla_{\mu} J^{\mu}=0, \quad J^{\mu}=\left(\beta G^{\mu \nu}-\gamma T^{\mu \nu}\right) \nabla_{\nu} \Psi .
$$

Then note that the current vector $J^{\mu}$ "contains" the metric field equations of the BBMB action (5.11). As such we can refer to action $S_{0}$ as being precursor of the higher order action $S_{1}$. We will see that this will enable to obtain our desired result even though the field equations associated to (5.10) are very complex. We give the field equations for the scalar field $\phi$ and the metric, in the appendix and let's denote them $\mathcal{E}_{\phi}=0$ and $\mathcal{H}_{\mu \nu}=0$ respectively.

We now proceed to adopt a spherically symmetric anzatz

$$
d s^{2}=-h(r) d t^{2}+\frac{d r^{2}}{f(r)}+r^{2} d \Omega^{2},
$$

where $d \Omega^{2}$ is the line element for the 2 -sphere, while for the scalar fields we set

$$
\phi=\phi(r) \quad \text { and } \quad \Psi=\Psi(t, r)=q t+\psi(r) .
$$

Assuming that

$$
\beta G_{r r}-\gamma T_{r r}=0
$$


kills the dangerous $J^{r}$ component of the current satisfying the regularity requirement of [16] without imposing a trivial $\psi$-field $[17,38]$. Equation $(5.17)$, is a solution to $\mathcal{H}_{t r}=0$. In fact the primary hair charge ${ }^{2}$ associated to the Galileon $\Psi$ is in this way set to zero. Now having an extra scalar, $\phi$ we may hope to keep the primary hair charge associated to that field. Indeed this is what happens; we solve (5.17) for the function $f(r)$,

$$
f(r)=\frac{\phi^{2}(r) h(r)\left(12 \beta-\gamma \phi^{2}(r)\right)}{12 \beta \phi^{2}(r)(r h(r))^{\prime}-\gamma(r \phi(r))^{\prime}\left(r \phi^{3}(r) h(r)\right)^{\prime}} .
$$

The above result for $f(r)$ satisfies the field equation of $\Psi$ and the flux equation $\mathcal{H}_{t r}=0$. Then we turn to $\mathcal{H}_{r r}=0$ and solve for $\psi^{\prime}(r)$,

$$
\psi^{\prime}= \pm \frac{\sqrt{q^{2}\left(-12 \beta+\gamma \phi^{2}\right) h^{\prime}\left(-12 \beta r+\frac{\gamma}{2}\left(\phi^{2} r^{2}\right)^{\prime}\right)-12(\gamma \zeta-\beta \eta)\left(\frac{\left(\phi^{2}\right)^{\prime}}{4}\left(h^{2} r^{2}\right)^{\prime}+3 h^{2} r \phi^{\prime}(r \phi)^{\prime}\right)}}{h\left(-12 \beta+\gamma \phi^{2}\right)} .
$$

One can attempt a brute force resolution of the final equation $\mathcal{H}_{t t}=0$ in order to obtain the metric component $h(r)$. We did not manage to solve this equation in all generality. Guided however by our previous form of the scalar field $\phi$, namely (5.3) and (5.4) we note that they simplify considerably (5.18). We choose therefore to impose the former $\phi=c_{0} / r$ for it is regular apart from $r=0$ whereas the latter is singular for $h=0$, the location of a possible event horizon. The integration constant $c_{0}$ is the scalar hair associated to $\phi$. We get that

$$
f(r)=\frac{h(r)\left(12 \beta r^{2}-\gamma c_{0}^{2}\right)}{12 \beta r^{2}(r h(r))^{\prime}}
$$

and

$$
\psi^{\prime}(r)=\mp q \frac{\sqrt{6 r(\gamma \zeta-\beta \eta) c_{0}^{2}\left(h^{2}(r) r^{2}\right)^{\prime}+\left(12 r^{2} \beta-\gamma c_{0}^{2}\right) h^{\prime}(r) 12 \beta r^{3}}}{h(r)\left(12 r^{2} \beta-\gamma c_{0}^{2}\right)} .
$$

Going back to the equation $\mathcal{H}_{t}^{t}=0$ and substituting all of the above expressions we see that setting,

$$
h(r)=-\frac{\mu}{r}+\frac{1}{r} \int \frac{k(r)}{12 r^{2} \beta-\gamma c_{0}^{2}} d r,
$$

then equation $\mathcal{H}_{t}{ }^{t}=0$ reduces to just an algebraic equation for $k(r)$,

$$
q^{2} \beta\left(-12 r^{2} \beta+\gamma c_{0}^{2}\right)^{2}+k(r)\left(-24 r^{2} \beta \zeta+(\gamma \zeta+\beta \eta) c_{0}^{2}\right)-C_{1} r k^{3 / 2}=0
$$

where $C_{1}$ is an integration constant. Any solution to the above cubic gives a solution to the full system of equation, retracing each field step by step. Generically, these solutions will not have usual asymptotics and will be quite tedious to write down.

\footnotetext{
${ }^{2}$ This is the integration constant emanating from the scalar equation (5.14).
} 
Let us therefore look at some particular solutions. Start by assuming $f=h$. This immediately leads us, using (5.23) to,

$$
\begin{aligned}
f(r)=h(r) & =1-\frac{m}{r}+\frac{\gamma c_{0}^{2}}{12 \beta r^{2}}, \\
\phi(r) & =\frac{c_{0}}{r}, \\
\psi^{\prime}(r) & = \pm q \frac{\sqrt{m r-\frac{\gamma c_{0}^{2}}{12 \beta}}}{r h(r)}, \\
\beta \eta+\gamma\left(q^{2} \beta-\zeta\right) & =0
\end{aligned}
$$

and $C_{1}^{2}=12 \beta\left(\beta q^{2}-2 \zeta\right)^{2}$. We see that we have a black hole solution with primary scalar charge $c_{0}$ playing a similar role to an EM gauge field for the metric solution. Going as before to EF coordinates (5.7) we see that,

$$
\psi=q v-q \int \frac{d r}{1 \pm \sqrt{1-h(r)}}
$$

which is regular at the outer event horizon $h\left(r_{h}\right)=0$ (once again only the plus branch of (5.28) is taken). We have therefore obtained a scalar tensor black hole which has primary hair charge $c_{0}$ for the scalar field $\phi$ while it has a regular scalar Galileon field $\psi$ with an additional charge $q$. The geometry of the black hole is similar to that of ReissnerNordstrom.

The second possibility we consider is to take $C_{1}=0$ in (5.23). For simplicity we impose $\beta \eta-\gamma \zeta=0$ and we get,

$$
h(r)=1-\frac{m}{r}, \quad f(r)=\left(1-\frac{m}{r}\right)\left(1-\frac{\gamma c_{0}^{2}}{12 \beta r^{2}}\right)
$$

and

$$
\psi^{\prime}=\mp \frac{q}{h(r)} \sqrt{\frac{m r}{r^{2}-\frac{\gamma c_{0}^{2}}{12 \beta}}} .
$$

Taking $\gamma \beta<0$ is enough-for example-to ensure that the only relevant zero of $h(r)$ and $f(r)$ is $r_{h}=m$. Otherwise we need $m>\sqrt{\frac{\gamma c_{0}^{2}}{12 \beta}}$. Then again, as before the EF chart, which in this case reads as

$$
v=t+\int \frac{d r}{\sqrt{f(r) h(r)}}
$$

is regular and we get,

$$
\psi=q v-q \int \frac{d r}{\sqrt{\left(1-\frac{\gamma c_{0}^{2}}{12 \beta r^{2}}\right)}\left(1 \mp \sqrt{\frac{m}{r}}\right)} .
$$

Again the plus branch is the acceptable one. 


\section{Conclusions}

In this paper we have studied certain bi-scalar-tensor theories and found hairy black hole solutions. The theories we have investigated are characterized by the property that part of the action is conformally invariant. As a result one scalar is a Galileon field with shift symmetry, while the latter is coupled conformally to part of the action. Our aim has been to combine older techniques developed for conformally coupled scalars [34, 35, 49-52] with newer ones [38] with the ultimate goal of finding black holes with primary hair. This was eventually achieved, in a step by step manner in the last section.

The bi-scalar tensor theories we studied emerge from the general scalar-tensor theory put forward by Hordenski [3]. Indeed taking one of the Horndeski scalar-tensor interaction terms $G(\Psi) G^{\mu \nu} \nabla_{\mu} \Psi \nabla_{\nu} \Psi$ and requiring conformal invariance with the help of an extra scalar field $\phi$ we constructed conformally invariant terms which yield second order field equations. Fixing the coupling $G(\Psi)$ in this way, our aim was to construct a theory where regular solutions may be found due to the underlying conformal symmetry much like in the case of the BBMB and MTZ solutions [34, 35, 49-52]. In order to do so, we had the freedom to choose the conformal weight of our fields without the need of adding canonical kinetic terms. These theories are a subset of the full general conformally invariant bi-scalar theories that have been recently introduced in [56].

Having fixed the higher order conformal term (2.16) with a particular simplifying conformal weight we added to the action an Einstein-Hilbert, a cosmological constant and an electromagnetic field breaking the conformal invariance for the full theory. Indeed, had we worked with a theory admitting conformal invariance the solutions would have had one free function as a result of the symmetry. We also considered the case of an additional conformal coupling of the second scalar $\phi$ in the manner of $[34,35]$. In all cases we found anti de Sitter planar black holes where the second scalar, akin in some cases to MTZ [4952], was everywhere regular apart from the black hole singularity. The Galileon field was shown to encounter problems on the horizon and within but we went on to show how this singularity could be eliminated by including linear time dependance in the manner of [38]. Motivated by the differing approaches of $[34,35]$ and [38] we then went on to consider a slightly different bi-scalar-tensor theory which invoked some of the ideas of both approaches. In this way we eventually succeeded in finding two asymptotically flat hairy scalar tensor black holes where both scalars are regular and furthermore the one associated to the BBMB action carries primary scalar hair.

The construction we have put together in the last section of this paper can be extended to other terms appearing in the Horndeski Lagrangian and this will allow to find differing black hole solutions of the Horndeski theory in a closed form. It would also be interesting to understand the role of the integration constant $q$ associated to time dependence of the scalar field. Is it a real charge? After all it is not the Galileon $\Psi$ that appears in the action, it is the derivative. In this sense it is also not clear that one should worry about regularity of the field $\Psi$ itself, one should maybe worry more about its derivative in the same way one does for the Maxwell field strength rather than the potential. A careful study of the underlying thermodynamic properties would also be interesting. This would 
most likely shed some light in the question of relevant and non relevant charges of the solutions and how they make compare to possibly GR solutions-if one can find a common thermodynamic bath.

\section{Acknowledgments}

We thank Jorge Zanelli for usefull discussions. Additionally we thank the participants of the "Meeting on the Horizon conference", held in Valparaiso for their comments during the presentation of various results of this project. C.C. is delighted to thank Eugeny Babichev and Mokhtar Hassaine for discussions. M.T. thanks the Laboratoire de Physique Théorique (LPT), Univ. Paris-Sud in Orray and the National Technical University of Athens (N.T.U.A.) for hospitality during various stages of this work. T.K. and E.P. thank the Centro de Estudios Cientificos (CECs) for hospitality during the initial and final stages of this work. MT was funded by the FONDECYT Grant No. 3120143. The Centro de Estudios Cientificos (CECs) is funded by the Chilean Government through the Centers of Excellence Base Financing Program of Conicyt. E.P is supported partially by ARISTEIA II action of the operational programme education and long life learning which is co-funded by the European Union (European Social Fund) and National Resources. T.K. was funded by the FONDECYT Grant No. 3140261.

\section{A Variations}

Varying (5.10) with respect to $\phi$ we get:

$$
\begin{aligned}
\mathcal{E}_{\phi}= & \eta\left(\square \phi-\frac{1}{6} R \phi\right) \\
& +\gamma\left[\nabla_{\nu}\left(\nabla^{\nu} \Psi \nabla_{\mu} \phi \nabla^{\mu} \Psi\right)-\frac{1}{2} \nabla^{\alpha}\left(\nabla_{\alpha} \phi \nabla_{\mu} \Psi \nabla^{\mu} \Psi\right)+\frac{1}{3} \nabla^{\alpha}\left(\nabla_{\alpha} \phi \nabla_{\mu} \Psi \nabla^{\mu} \Psi\right)-\frac{1}{6} \square \phi \nabla_{\mu} \Psi \nabla^{\mu} \Psi\right. \\
& -\frac{1}{3} \nabla_{\mu}\left(\nabla^{\mu} \Psi \nabla_{\nu} \phi \nabla^{\nu} \Psi\right)+\frac{1}{6} \nabla_{\mu} \nabla_{\nu} \phi \nabla^{\mu} \Psi \nabla^{\nu} \Psi \\
& -\frac{1}{6} \phi G_{\mu \nu} \nabla^{\mu} \Psi \nabla^{\nu} \Psi+\frac{1}{6} \nabla_{\nu}\left(\nabla^{\nu} \Psi \nabla_{\mu} \phi \nabla^{\mu} \Psi\right)+\frac{1}{6} \square \Psi \nabla_{\nu} \phi \nabla^{\nu} \Psi+\frac{1}{6} \phi(\square \Psi)^{2} \\
& +\frac{1}{6} \nabla_{\nu} \phi \nabla^{\mu} \Psi \nabla_{\mu} \nabla^{\nu} \Psi+\frac{1}{6} \phi \nabla_{\mu} \nabla_{\nu} \Psi \nabla^{\mu} \nabla^{\nu} \Psi-\frac{1}{6} \nabla^{\alpha}\left(\nabla_{\alpha} \phi \nabla_{\mu} \Psi \nabla^{\mu} \Psi\right)-\frac{1}{3} \nabla_{\mu} \phi \nabla_{\nu} \Psi \nabla^{\mu} \nabla^{\nu} \Psi \\
& \left.-\frac{1}{3} \phi \nabla_{\mu} \nabla_{\nu} \Psi \nabla^{\mu} \nabla^{\nu} \Psi-\frac{1}{6} \phi R_{\mu \nu} \nabla^{\mu} \Psi \nabla^{\nu} \Psi\right]=0,
\end{aligned}
$$

where when collecting similar terms, the above expression is written as

$$
\begin{aligned}
\mathcal{E}_{\phi}= & \eta\left(\square \phi-\frac{1}{6} R \phi\right) \\
& +\gamma\left[\frac{5}{6} \nabla_{\nu}\left(\nabla^{\nu} \Psi \nabla_{\mu} \phi \nabla^{\mu} \Psi\right)-\frac{1}{3} \nabla^{\alpha}\left(\nabla_{\alpha} \phi \nabla_{\mu} \Psi \nabla^{\mu} \Psi\right)-\frac{1}{6} \square \phi \nabla_{\mu} \Psi \nabla^{\mu} \Psi+\frac{1}{6} \nabla_{\mu} \nabla_{\nu} \phi \nabla^{\mu} \Psi \nabla^{\nu} \Psi\right. \\
& -\frac{1}{6} \phi G_{\mu \nu} \nabla^{\mu} \Psi \nabla^{\nu} \Psi+\frac{1}{6} \square \Psi \nabla_{\nu} \phi \nabla^{\nu} \Psi+\frac{1}{6} \phi(\square \Psi)^{2}-\frac{1}{6} \nabla_{\nu} \phi \nabla^{\mu} \Psi \nabla_{\mu} \nabla^{\nu} \Psi \\
& \left.-\frac{1}{6} \phi \nabla_{\mu} \nabla_{\nu} \Psi \nabla^{\mu} \nabla^{\nu} \Psi-\frac{1}{6} \phi R_{\mu \nu} \nabla^{\mu} \Psi \nabla^{\nu} \Psi\right]=0
\end{aligned}
$$


The metric field equations are

$$
\begin{aligned}
\mathcal{H}_{\mu \nu}= & \zeta G_{\mu \nu}-\eta\left(\frac{1}{2} \nabla_{\mu} \phi \nabla_{\nu} \phi-\frac{1}{4} g_{\mu \nu} \nabla_{\alpha} \phi \nabla^{\alpha} \phi+\frac{1}{12}\left(g_{\mu \nu} \square-\nabla_{\mu} \nabla_{\nu}+G_{\mu \nu}\right) \phi^{2}\right) \\
& +\beta\left(-\frac{1}{2} g_{\mu \nu} G^{\alpha \beta} \nabla_{\alpha} \Psi \nabla_{\beta} \Psi+2 G_{(\mu}{ }^{\lambda} \nabla_{\nu)} \Psi \nabla_{\lambda} \Psi+\frac{1}{2} R \nabla_{\mu} \Psi \nabla_{\nu} \Psi-\frac{1}{2} R_{\mu \nu} \nabla^{\alpha} \Psi \nabla_{\alpha} \Psi+\frac{1}{2} g_{\mu \nu}\left((\square \Psi)^{2}\right.\right. \\
& \left.\left.-\nabla_{\alpha} \nabla_{\beta} \Psi \nabla^{\alpha} \nabla^{\beta} \Psi-R_{\alpha \beta} \nabla^{\alpha} \Psi \nabla^{\beta} \Psi\right)+\nabla_{\mu} \nabla^{\alpha} \Psi \nabla_{\nu} \nabla_{\alpha} \Psi-\square \Psi \nabla_{\mu} \nabla_{\nu} \Psi+R_{\mu \nu}^{\alpha}{ }_{\nu} \nabla_{\alpha} \Psi \nabla_{\beta} \Psi\right) \\
& +\gamma\left[\frac{1}{4} g_{\mu \nu} \nabla_{\alpha} \phi \nabla_{\beta} \phi \nabla^{\alpha} \Psi \nabla^{\beta} \Psi-\nabla_{(\mu} \phi \nabla_{\nu)} \Psi \nabla_{\alpha} \phi \nabla^{\alpha} \Psi-\frac{1}{8} g_{\mu \nu} \nabla_{\alpha} \phi \nabla^{\alpha} \phi \nabla_{\beta} \Psi \nabla^{\beta} \Psi\right. \\
& +\frac{1}{4} \nabla_{\mu} \phi \nabla_{\nu} \phi \nabla_{\beta} \Psi \nabla^{\beta} \Psi+\frac{1}{4} \nabla_{\alpha} \phi \nabla^{\alpha} \phi \nabla_{\mu} \Psi \nabla_{\nu} \Psi+\frac{1}{24} g_{\mu \nu} \square\left(\phi^{2}\right) \nabla_{\alpha} \Psi \nabla^{\alpha} \Psi-\frac{1}{12} \nabla_{\mu} \nabla_{\nu} \phi^{2} \nabla_{\alpha} \Psi \nabla^{\alpha} \Psi \\
& +\frac{1}{12} \nabla_{(\mu}\left(\nabla_{\nu)}\left(\phi^{2}\right) \nabla_{\alpha} \Psi \nabla^{\alpha} \Psi\right)-\frac{1}{24} g_{\mu \nu} \nabla^{\beta}\left(\nabla_{\beta} \phi^{2} \nabla_{\alpha} \Psi \nabla^{\alpha} \Psi\right)-\frac{1}{12} \square\left(\phi^{2}\right) \nabla_{\mu} \Psi \nabla_{\nu} \Psi \\
& -\frac{1}{24} g_{\mu \nu} \nabla_{\alpha} \nabla_{\beta} \phi^{2} \nabla^{\alpha} \Psi \nabla^{\beta} \Psi+\frac{1}{6} \nabla_{\alpha} \nabla_{(\mu} \phi^{2} \nabla_{\nu)} \Psi \nabla^{\alpha} \Psi-\frac{1}{12} \nabla_{\alpha}\left(\nabla_{(\mu} \phi^{2} \nabla_{\nu)} \Psi \nabla^{\alpha} \Psi\right) \\
& +\frac{1}{24} \nabla_{\alpha}\left(\nabla^{\alpha} \phi^{2} \nabla_{\mu} \Psi \nabla_{\nu} \Psi\right)+\frac{1}{24} \phi^{2} g_{\mu \nu} G^{\alpha \beta} \nabla_{\alpha} \Psi \nabla_{\beta} \Psi-\frac{1}{6} \phi^{2} G_{(\mu}{ }^{\lambda} \nabla_{\nu)} \Psi \nabla_{\lambda} \Psi-\frac{1}{24} \phi^{2} R \nabla_{\mu} \Psi \nabla_{\nu} \Psi \\
& +\frac{1}{24} \phi^{2} R_{\mu \nu} \nabla^{\alpha} \Psi \nabla_{\alpha} \Psi+\frac{1}{12} \nabla_{\alpha} \nabla_{(\mu}\left(\phi^{2}\right) \nabla^{\alpha} \Psi \nabla_{\nu)} \Psi+\frac{1}{12} \square \Psi \nabla_{(\mu}\left(\phi^{2}\right) \nabla_{\nu)} \Psi \\
& -\frac{1}{12} \nabla^{\alpha} \Psi \nabla_{(\mu}\left(\phi^{2}\right) \nabla_{\nu)} \nabla_{\alpha} \Psi-\frac{1}{12} \nabla_{\alpha}\left(\phi^{2}\right) \nabla_{(\mu} \nabla^{\alpha} \Psi \nabla_{\nu)} \Psi+\frac{1}{12} \nabla_{\alpha}\left(\phi^{2}\right) \nabla^{\alpha} \Psi \nabla_{\mu} \nabla_{\nu} \Psi \\
& -\frac{1}{24} \square\left(\phi^{2}\right) \nabla_{\mu} \Psi \nabla_{\nu} \Psi-\frac{1}{24} \nabla_{\mu} \nabla_{\nu}\left(\phi^{2}\right) \nabla^{\alpha} \Psi \nabla_{\alpha} \Psi-\frac{1}{24} g_{\mu \nu} \nabla_{\alpha} \nabla_{\beta}\left(\phi^{2}\right) \nabla^{\alpha} \Psi \nabla^{\beta} \Psi \\
& -\frac{1}{12} g_{\mu \nu} \nabla_{\alpha}\left(\phi^{2}\right) \nabla^{\alpha} \Psi \square \Psi+\frac{1}{12} g_{\mu \nu} \nabla_{\alpha}\left(\phi^{2}\right) \nabla_{\beta} \Psi \nabla^{\alpha} \nabla^{\beta} \Psi+\frac{1}{24} g_{\mu \nu} \square\left(\phi^{2}\right) \nabla^{\alpha} \Psi \nabla_{\alpha} \Psi \\
& -\frac{1}{12} \phi^{2}\left(\frac{1}{2} g_{\mu \nu}\left((\square \Psi)^{2}-\nabla_{\alpha} \nabla_{\beta} \Psi \nabla^{\alpha} \nabla^{\beta} \Psi-R_{\alpha \beta} \nabla^{\alpha} \Psi \nabla^{\beta} \Psi\right)+\nabla_{\mu} \nabla^{\alpha} \Psi \nabla_{\nu} \nabla_{\alpha} \Psi\right. \\
& \left.\left.-\square \Psi \nabla_{\mu} \nabla_{\nu} \Psi+R_{\mu}{ }_{\nu}{ }_{\nu} \nabla_{\alpha} \Psi \nabla_{\beta} \Psi\right)\right]=0 .
\end{aligned}
$$

Open Access. This article is distributed under the terms of the Creative Commons Attribution License (CC-BY 4.0), which permits any use, distribution and reproduction in any medium, provided the original author(s) and source are credited.

\section{References}

[1] R. Amanullah et al., Spectra and Light Curves of Six Type Ia Supernovae at $0.511<z<1.12$ and the Union2 Compilation, Astrophys. J. 716 (2010) 712 [arXiv:1004.1711] [INSPIRE].

[2] WMAP collaboration, G. Hinshaw et al., Nine-Year Wilkinson Microwave Anisotropy Probe (WMAP) Observations: Cosmological Parameter Results, Astrophys. J. Suppl. 208 (2013) 19 [arXiv: 1212.5226] [INSPIRE].

[3] G.W. Horndeski, Second-order scalar-tensor field equations in a four-dimensional space, Int. J. Theor. Phys. 10 (1974) 363 [InSPIRE].

[4] A. Nicolis, R. Rattazzi and E. Trincherini, The Galileon as a local modification of gravity, Phys. Rev. D 79 (2009) 064036 [arXiv:0811.2197] [inSPIRE].

[5] C. Deffayet, G. Esposito-Farese and A. Vikman, Covariant Galileon, Phys. Rev. D 79 (2009) 084003 [arXiv:0901.1314] [INSPIRE]. 
[6] C. Deffayet, S. Deser and G. Esposito-Farese, Generalized Galileons: All scalar models whose curved background extensions maintain second-order field equations and stress-tensors, Phys. Rev. D 80 (2009) 064015 [arXiv:0906.1967] [INSPIRE].

[7] C. Deffayet, X. Gao, D.A. Steer and G. Zahariade, From k-essence to generalised Galileons, Phys. Rev. D 84 (2011) 064039 [arXiv:1103.3260] [InSPIRE].

[8] N. Deruelle and J. Madore, On the quasilinearity of the Einstein-'Gauss-Bonnet' gravity field equations, gr-qc/0305004 [INSPIRE].

[9] C. Charmousis, Higher order gravity theories and their black hole solutions, Lect. Notes Phys. 769 (2009) 299 [arXiv:0805. 0568] [INSPIRE].

[10] C. Garraffo and G. Giribet, The Lovelock Black Holes, Mod. Phys. Lett. A 23 (2008) 1801 [arXiv:0805.3575] [INSPIRE].

[11] F. Mueller-Hoissen, Gravity Actions, Boundary Terms and Second Order Field Equations, Nucl. Phys. B 337 (1990) 709 [inSPIRE].

[12] F. Mueller-Hoissen, Nonminimal Coupling From Dimensional Reduction of the Gauss-Bonnet Action, Phys. Lett. B 201 (1988) 325 [INSPIRE].

[13] F. Mueller-Hoissen, Modification of Einstein Yang-Mills Theory From Dimensional Reduction of the Gauss-Bonnet Action, Class. Quant. Grav. 5 (1988) L35 [inSPIRE].

[14] K. Van Acoleyen and J. Van Doorsselaere, Galileons from Lovelock actions, Phys. Rev. D 83 (2011) 084025 [arXiv: 1102.0487] [InSPIRE].

[15] C. Charmousis, B. Gouteraux and E. Kiritsis, Higher-derivative scalar-vector-tensor theories: black holes, Galileons, singularity cloaking and holography, JHEP 09 (2012) 011 [arXiv: 1206.1499] [INSPIRE].

[16] L. Hui and A. Nicolis, No-Hair Theorem for the Galileon, Phys. Rev. Lett. 110 (2013) 241104 [arXiv:1202.1296] [INSPIRE].

[17] T.P. Sotiriou and S.-Y. Zhou, Black hole hair in generalized scalar-tensor gravity, Phys. Rev. Lett. 112 (2014) 251102 [arXiv:1312.3622] [INSPIRE].

[18] C. Germani, L. Martucci and P. Moyassari, Introducing the Slotheon: a slow Galileon scalar field in curved space-time, Phys. Rev. D 85 (2012) 103501 [arXiv:1108.1406] [INSPIRE].

[19] C. Charmousis, E.J. Copeland, A. Padilla and P.M. Saffin, General second order scalar-tensor theory, self tuning and the Fab Four, Phys. Rev. Lett. 108 (2012) 051101 [arXiv:1106.2000] [INSPIRE].

[20] C. Charmousis, E.J. Copeland, A. Padilla and P.M. Saffin, Self-tuning and the derivation of a class of scalar-tensor theories, Phys. Rev. D 85 (2012) 104040 [arXiv:1112.4866] [INSPIRE].

[21] L. Amendola, Cosmology with nonminimal derivative couplings, Phys. Lett. B 301 (1993) 175 [gr-qc/9302010] [INSPIRE].

[22] L. Amendola, C. Charmousis and S.C. Davis, Constraints on Gauss-Bonnet gravity in dark energy cosmologies, JCAP 12 (2006) 020 [hep-th/0506137] [INSPIRE].

[23] S.V. Sushkov, Exact cosmological solutions with nonminimal derivative coupling, Phys. Rev. D 80 (2009) 103505 [arXiv:0910.0980] [INSPIRE].

[24] C. Germani and A. Kehagias, UV-Protected Inflation, Phys. Rev. Lett. 106 (2011) 161302 [arXiv: 1012.0853] [INSPIRE]. 
[25] G. Koutsoumbas, K. Ntrekis and E. Papantonopoulos, Gravitational Particle Production in Gravity Theories with Non-minimal Derivative Couplings, JCAP 08 (2013) 027 [arXiv: 1305.5741] [INSPIRE].

[26] C. Gao, When scalar field is kinetically coupled to the Einstein tensor, JCAP 06 (2010) 023 [arXiv: 1002.4035] [INSPIRE].

[27] L.N. Granda, Non-minimal Kinetic coupling to gravity and accelerated expansion, JCAP 07 (2010) 006 [arXiv:0911.3702] [INSPIRE].

[28] E.N. Saridakis and S.V. Sushkov, Quintessence and phantom cosmology with non-minimal derivative coupling, Phys. Rev. D 81 (2010) 083510 [arXiv:1002.3478] [INSPIRE].

[29] M. Rinaldi, Black holes with non-minimal derivative coupling, Phys. Rev. D 86 (2012) 084048 [arXiv: 1208.0103] [INSPIRE].

[30] A. Anabalon, A. Cisterna and J. Oliva, Asymptotically locally AdS and flat black holes in Horndeski theory, Phys. Rev. D 89 (2014) 084050 [arXiv:1312.3597] [INSPIRE].

[31] M. Minamitsuji, Solutions in the scalar-tensor theory with nonminimal derivative coupling, Phys. Rev. D 89 (2014) 064017 [arXiv: 1312.3759] [INSPIRE].

[32] M. Minamitsuji, Braneworlds with field derivative coupling to the Einstein tensor, Phys. Rev. D 89 (2014) 064025 [arXiv:1312.3760] [INSPIRE].

[33] A. Cisterna and C. Erices, Asymptotically locally AdS and flat black holes in the presence of an electric field in the Horndeski scenario, Phys. Rev. D 89 (2014) 084038 [arXiv: 1401.4479] [INSPIRE].

[34] N.M. Bocharova, K.A. Bronnikov and V.N. Melnikov, An exact solution of the system of einstein equations and mass-free scalar field, Vestn. Mosk. Univ. Fiz. Astro. 6 (1970) 706.

[35] J.D. Bekenstein, Exact solutions of Einstein conformal scalar equations, Annals Phys. 82 (1974) 535 [inSPIRE].

[36] T. Kolyvaris, G. Koutsoumbas, E. Papantonopoulos and G. Siopsis, Scalar Hair from a Derivative Coupling of a Scalar Field to the Einstein Tensor, Class. Quant. Grav. 29 (2012) 205011 [arXiv:1111. 0263] [INSPIRE].

[37] T. Kolyvaris, G. Koutsoumbas, E. Papantonopoulos and G. Siopsis, Phase Transition to a Hairy Black Hole in Asymptotically Flat Spacetime, JHEP 11 (2013) 133 [arXiv:1308.5280] [INSPIRE].

[38] E. Babichev and C. Charmousis, Dressing a black hole with a time-dependent Galileon, arXiv: 1312.3204 [INSPIRE].

[39] T. Kobayashi and N. Tanahashi, Exact black hole solutions in shift symmetric scalar-tensor theories, arXiv:1403.4364 [INSPIRE].

[40] I. Bars, S.-H. Chen and N. Turok, Geodesically Complete Analytic Solutions for a Cyclic Universe, Phys. Rev. D 84 (2011) 083513 [arXiv:1105.3606] [InSPIRE].

[41] I. Bars, S.-H. Chen, P.J. Steinhardt and N. Turok, Antigravity and the Big Crunch/Big Bang Transition, Phys. Lett. B 715 (2012) 278 [arXiv:1112.2470] [INSPIRE].

[42] I. Bars, S.-H. Chen, P.J. Steinhardt and N. Turok, Complete Set of Homogeneous Isotropic Analytic Solutions in Scalar-Tensor Cosmology with Radiation and Curvature, Phys. Rev. D 86 (2012) 083542 [arXiv:1207.1940] [INSPIRE]. 
[43] I. Bars, Traversing Cosmological Singularities, Complete Journeys Through Spacetime Including Antigravity, arXiv:1209.1068 [INSPIRE].

[44] I. Bars, P. Steinhardt and N. Turok, Local Conformal Symmetry in Physics and Cosmology, Phys. Rev. D 89 (2014) 043515 [arXiv: 1307.1848] [INSPIRE].

[45] J.J.M. Carrasco, W. Chemissany and R. Kallosh, Journeys Through Antigravity?, JHEP 01 (2014) 130 [arXiv:1311.3671] [INSPIRE].

[46] G. 't Hooft, Probing the small distance structure of canonical quantum gravity using the conformal group, arXiv:1009.0669 [INSPIRE].

[47] O. Mišković and R. Olea, Topological regularization and self-duality in four-dimensional anti-de Sitter gravity, Phys. Rev. D 79 (2009) 124020 [arXiv:0902.2082] [INSPIRE].

[48] J. Maldacena, Einstein Gravity from Conformal Gravity, arXiv:1105.5632 [INSPIRE].

[49] C. Martinez, R. Troncoso and J. Zanelli, Exact black hole solution with a minimally coupled scalar field, Phys. Rev. D 70 (2004) 084035 [hep-th/0406111] [INSPIRE].

[50] C. Martinez, J.P. Staforelli and R. Troncoso, Topological black holes dressed with a conformally coupled scalar field and electric charge, Phys. Rev. D 74 (2006) 044028 [hep-th/0512022] [INSPIRE].

[51] C. Martinez and R. Troncoso, Electrically charged black hole with scalar hair, Phys. Rev. D 74 (2006) 064007 [hep-th/0606130] [INSPIRE].

[52] C. Martinez, R. Troncoso and J. Zanelli, De Sitter black hole with a conformally coupled scalar field in four-dimensions, Phys. Rev. D 67 (2003) 024008 [hep-th/0205319] [INSPIRE].

[53] A. Anabalon and A. Cisterna, Asymptotically (anti) de Sitter Black Holes and Wormholes with a Self Interacting Scalar Field in Four Dimensions, Phys. Rev. D 85 (2012) 084035 [arXiv:1201.2008] [INSPIRE].

[54] P.A. González, E. Papantonopoulos, J. Saavedra and Y. Vásquez, Four-Dimensional Asymptotically AdS Black Holes with Scalar Hair, JHEP 12 (2013) 021 [arXiv:1309.2161] [INSPIRE].

[55] T. Kobayashi and N. Tanahashi, Exact black hole solutions in shift symmetric scalar-tensor theories, arXiv:1403.4364 [INSPIRE].

[56] A. Padilla, D. Stefanyszyn and M. Tsoukalas, Generalised Scale Invariant Theories, Phys. Rev. D 89 (2014) 065009 [arXiv: 1312.0975] [INSPIRE].

[57] S. Deser, Scale Invariance and Gravitational Coupling, Ann. Phys. 59 (1970) 248.

[58] R. Troncoso and M. Tsoukalas, Conformally Coupled Scalar Fields in Diverse Dimensions: Nontrivial Vacua, Instantons and a Generalization of the Yamabe problem, CECS-PHY-11/11.

[59] A. Padilla and V. Sivanesan, Covariant multi-galileons and their generalisation, JHEP 04 (2013) 032 [arXiv:1210.4026] [INSPIRE].

[60] T. Kobayashi, N. Tanahashi and M. Yamaguchi, Multifield extension of G inflation, Phys. Rev. D 88 (2013) 083504 [arXiv:1308.4798] [INSPIRE].

[61] V. Sivanesan, Proof of the most general multiple-scalar field theory in Minkowski space-time free of Ostrogradski Ghost, arXiv:1307.8081 [INSPIRE]. 
[62] A. Iorio, L. O'Raifeartaigh, I. Sachs and C. Wiesendanger, Weyl gauging and conformal invariance, Nucl. Phys. B 495 (1997) 433 [hep-th/9607110] [InSPIRE].

[63] S. Faci, Constructing conformally invariant equations by using Weyl geometry, Class. Quant. Grav. 30 (2013) 115005 [arXiv:1212.2599] [INSPIRE].

[64] J. Oliva and S. Ray, Conformal couplings of a scalar field to higher curvature terms, Class. Quant. Grav. 29 (2012) 205008 [arXiv:1112.4112] [INSPIRE].

[65] Y. Bardoux, M.M. Caldarelli and C. Charmousis, Conformally coupled scalar black holes admit a flat horizon due to axionic charge, JHEP 09 (2012) 008 [arXiv:1205.4025] [INSPIRE].

[66] M.M. Caldarelli, C. Charmousis and M. Hassaïne, AdS black holes with arbitrary scalar coupling, JHEP 10 (2013) 015 [arXiv:1307.5063] [INSPIRE].

[67] E. Babichev, Galileon accretion, Phys. Rev. D 83 (2011) 024008 [arXiv:1009.2921] [INSPIRE].

[68] E. Babichev, C. Deffayet and G. Esposito-Farese, Constraints on Shift-Symmetric Scalar-Tensor Theories with a Vainshtein Mechanism from Bounds on the Time Variation of G, Phys. Rev. Lett. 107 (2011) 251102 [arXiv:1107.1569] [INSPIRE].

[69] E. Babichev and G. Esposito-Farese, Time-Dependent Spherically Symmetric Covariant Galileons, Phys. Rev. D 87 (2013) 044032 [arXiv:1212.1394] [INSPIRE]. 